\title{
Transforming acidic coiled-coil 3 and Aurora-A interact in human thyrocytes and their expression is deregulated in thyroid cancer tissues
}

\author{
Salvatore Ulisse, Enke Baldini, Matteo Toller ${ }^{1}$, Jean-Guy Delcros ${ }^{2}$, \\ Aurélie Guého ${ }^{2}$, Francesco Curcio ${ }^{1}$, Enrico De Antoni ${ }^{3}$, Laura Giacomelli ${ }^{3}$, \\ Francesco S Ambesi-Impiombato ${ }^{1}$, Sarah Bocchini, Massimino D'Armiento and \\ Yannick Arlot-Bonnemains ${ }^{2}$
}

\author{
Department of Experimental Medicine, University of Rome 'La Sapienza', Rome, Italy \\ ${ }^{1}$ Department of Pathology and Experimental Medicine and Clinic, University of Udine, Udine, Italy \\ ${ }^{2}$ Cycle Cellulaire et Pharmacologie, CNRS -UMR 6061 'Génétique et Dévelopement', IFR 140 G F A S, Faculté de Médecine, \\ Université de Rennes 1, 2 Avenue du Pr Léon Bernard, CS 34317, 35043 Rennes Cedex, France \\ ${ }^{3}$ Department of Surgical Sciences, University of Rome 'La Sapienza', Rome, Italy \\ (Correspondence should be addressed to Y Arlot-Bonnemains; Email: yannick.arlot@univ-rennes1.fr)
}

\begin{abstract}
Aurora-A kinase has recently been shown to be deregulated in thyroid cancer cells and tissues. Among the Aurora-A substrates identified, transforming acidic coiled-coil (TACC3), a member of the TACC family, plays an important role in cell cycle progression and alterations of its expression occur in different cancer tissues. In this study, we demonstrated the expression of the TACC3 gene in normal human thyroid cells (HTU5), and its modulation at both mRNA and protein levels during cell cycle. Its expression was found, with respect to HTU5 cells, unchanged in cells derived from a benign thyroid follicular tumor (HTU42), and significantly reduced in cell lines derived from follicular (FTC-133), papillary (B-CPAP), and anaplastic thyroid carcinomas (CAL-62 and 8305C). Moreover, in 16 differentiated thyroid cancer tissues, TACC3 mRNA levels were found, with respect to normal matched tissues, reduced by twofold in $56 \%$ of cases and increased by twofold in $44 \%$ of cases. In the same tissues, a correlation between the expression of the TACC 3 and Aurora-A mRNAs was observed. TACC3 and Aurora-A interact in vivo in thyroid cells and both proteins localized onto the mitotic structure of thyroid cells. Finally, TACC3 localization on spindle microtubule was no more observed following the inhibition of Aurora kinase activity by VX-680. We propose that Aurora-A and TACC3 interaction is important to control the mitotic spindle organization required for proper chromosome segregation.
\end{abstract}

Endocrine-Related Cancer (2007) 14 827-837

\section{Introduction}

The transforming acidic coiled-coil (TACC) proteins form an evolutionarily conserved family characterized by the presence at their C-terminal end of a common coiled-coil motif of $\sim 200$ amino acids (Gergely 2002, Still et al. 2004). In human, three members TACC1, TACC2, and TACC 3 have been identified and they are involved in the progression of mitosis (Sadek et al. 2000, Gergely 2002). The relevance of TACC3 physiological function has been assessed by TACC3 knockout mice, which die during embryogenesis due to a massive increase in apoptosis and overexpression of the p53 target gene (Piekorz et al. 2002, Sadek et al. 2003). TACC 3 protein is localized around the centrosomes during mitosis where it forms complexes with the microtubule-associated protein ch-TOG following TACC3 phosphorylation by Aurora-A kinase (Gergely et al. 2000, Gergely 2002, Pascreau et al. 2005). The latter belongs to the Aurora kinase family which includes three members, namely 
Aurora-A, Aurora-B, and Aurora-C (Bischoff \& Plowman 1999, Carmena \& Earnshaw 2003). AuroraA is mainly associated with centrosome in mitotic cells where it interacts with and phosphorylates several substrates including Eg5, TPX2, and the abovedescribed TACC3 involved in spindle formation and stability (Bischoff \& Plowman 1999, Arlot-Bonnemains \& Prigent 2002). Aurora-A has been shown to be overexpressed in solid tumor and its increase correlates with centrosome amplification (Bischoff \& Plowman 1999). Indeed, regulation of Aurora-A gene expression is critical for centrosome functions and its up- or downregulation leads to abnormal mitosis as well as to chromosome segregation and cytokinesis defects (Bischoff \& Plowman 1999). There is also evidence that $T A C C$ genes expression is deregulated in different cancer tissues. In fact, TACCI gene was first discovered as a gene amplified in breast cancer and its overexpression causes fibroblast transformation, suggesting that its amplification contributes to cancer progression (Still et al. 1999b). However, a reduction in its expression has been documented in ovarian and breast cancer tissues (Conte et al. 2002, Lauffart et al. 2005). Similarly, TACC 2 and TACC 3 expression has been shown to be reduced in several cancers (Chen et al. 2000, Lauffart et al. 2003, Lauffart et al. 2005). Moreover, a number of malignant tumors exhibit deletions or rearrangements of $4 \mathrm{p} 16$ and $8 \mathrm{p} 11$, the loci of TACC3 and TACC1 genes respectively (Sato et al. 1991, Still et al. 1999a).

Thyroid follicular cell-derived cancers are often characterized by chromosomal instability and aneuploidy (Shahedian et al. 2001, Ouyang et al. 2002). They represent the most common endocrine malignancy accounting for $1 \%$ of all new malignant diseases (Sherman 2003). Although derived from the same cell type, different thyroid neoplasms show specific histological features, biological behavior, and degree of differentiation, as a consequence of different genetic alterations (Shahedian et al. 2001, Ouyang et al. 2002, Nikiforova et al. 2003). The large majority of follicular thyroid cancers are represented by the differentiated papillary (B-CPAP) and follicular thyroid carcinomas (FTCs) which, following dedifferentiation, are thought to give rise to the aggressive anaplastic thyroid carcinomas (Kinder 2003, Pasieka 2003).

We recently demonstrated an altered expression of the Aurora-A gene in cell lines derived from different histotypes of human thyroid tumors and in papillary carcinoma tissues (Ulisse et al. 2006a). Since the TACC 3 protein represents a substrate of Aurora-A kinase and, to the best of our knowledge, the expression of the three TACC genes has never been characterized in human thyroid tissues, we here investigated the TACC3 expression, the cellular localization in normal and transformed human thyrocytes, and its interaction with Aurora-A. Finally, we compared the expression level of TACC 3 and AuroraA in thyroid cancer tissues.

\section{Materials and methods}

\section{Cell lines and materials}

Dulbecco's modified Eagle's medium, RPMI 1640 medium, ham's medium nutrient mixture F-12, PBS, fetal bovine serum (FBS), trypsin, EDTA, PBS, L-glutamine $100 \times(200 \mathrm{mM})$, and penicillin/streptomycin solution $100 \times$ were purchased from EuroClone (Paignton-Devon, UK). The Aurora kinase inhibitor VX-680 was obtained from KAWA Technology (San Diego, CA, USA). Oligo(dT) ${ }_{12-18}$ primer, Trizol, dNTP mix, and M-MLV reverse transcriptase were purchased from Invitrogen. HotMaster Taq DNA polymerase and Perfectprep Gel Cleanup Kit were obtained from Eppendorf (Hamburg, Germany). All primers were from PRIMM (Milan, Italy) and $100 \mathrm{bp}$ DNA ladder from New England BioLabs (Beverly, MA, USA). Proteases inhibitors were purchased from Roche, 4-(2-amino-ethyl) benzenesulfonyl fluoride hydrochloride, sodium orthovanadate, sodium pyrophosphate, the rabbit polyclonal anti-actin antibody, the anti- $\beta$-tubulin, and the anti- $\gamma$-tubulin monoclonal antibodies were obtained from Sigma Chemical Co. The goat (sc-5885) and the rabbit (sc-22773) polyclonal anti-TACC3 antibodies were purchased from Santa Cruz (Cambridge, UK). The monoclonal antiAurora-A antibody (clone 35C1) was obtained from Abcam (Paris, France). All secondary antibodies horseradish peroxidase conjugated were purchased from Jackson Immuno Research Laboratories (Baltimore, MD, USA).

\section{Cell cultures and human thyroid tissues}

The normal strain of human thyrocytes (HTU5) has been cultured as previously described (Curcio et al. 1994, Perrella et al. 1997). These diploid and nontumorigenic cells retain in culture the functional feature of normal human thyrocytes, such as the ability to express the major thyroid specific genes (i.e. thyroglobulin and thyrotrophin (TSH) receptor) and to increase cAMP level following TSH stimulation. All the other tumor-derived cell lines have been cultured as previously described (Ulisse et al. 2006a). The cells were maintained in continuous monolayer cultures at $37{ }^{\circ} \mathrm{C}$ and $5 \% \mathrm{CO}_{2}$, expanded up to $70-80 \%$ 
confluence and then employed for the experiments as described below. To investigate the effects of serum on normal human thyrocyte proliferation and TACC 3 expression, cells have been cultured for 4 days in the presence of 5 or $0.3 \%$ FBS. Following 4 days in medium containing low serum concentration, some of the cells have been treated for additional $24 \mathrm{~h}$ in medium containing 5\% FBS. The cells have been then processed to prepare total RNA and protein extracts.

Fragments of normal and tumoral thyroid tissues were obtained from surgical specimens of 16 female patients (age ranging from 36 to 76 years) of which 13 affected by papillary (papillary thyroid cancer, PTC, six follicular and seven classical variants) and 3 by FTC. Tissue samples were immediately frozen in liquid nitrogen, stored at $-80{ }^{\circ} \mathrm{C}$, and then used to prepare total RNA as described below.

\section{RNA isolation and analysis}

Total cellular RNA was extracted from the different cell lines by the acid guanidinium thiocyanate-phenolchloroform method (Chomczynski \& Sacchi 1987). The same protocol was used to obtain total RNA from normal and tumoral human thyroid tissues following homogenization of the samples by Ultra-Turrax in guanidinium thiocyanate. Five micrograms of total RNA samples were reverse transcribed and the obtained cDNAs used as a template for the quantitative PCR amplifications of TACC 3 , Aurora-A, and $\beta$-actin as internal control, using the following primers: TACC 3 , forward $5^{\prime}$-GAACTGGGGAAGATCATG GA-3' (exon 10/11), reverse 5'-CTCTTCGTTC TTGCGGTAGC-3' (exon 12/13), amplicon 222 bp; $\beta$-actin, forward $5^{\prime}$-CAAGAGATGGCCACGGCT GCT-3' (exon 3), reverse 5'-TCCTTCTGCATCCT GTCGGCA-3' (exon 4), amplicon 275 bp; Aurora A, forward $5^{\prime}$-CTGCATTTCAGGACCTGTTAAGG- $3^{\prime}$ (exon 1), reverse $5^{\prime}$-AACGCGCTGGGAAGAATTT$3^{\prime}$ (exon 2), amplicon $150 \mathrm{bp}$. Controls for DNA contamination were performed omitting the reverse transcriptase. Real-time PCR assay was performed on the LightCycler instrument (Roche Diagnostics), employing the FastStart DNA Master SYBR Green I Kit (Roche Applied Science) as previously described (Ulisse et al. 2006b). The PCR products were analyzed on $2 \%$ agarose gel and to determine the specificities of amplified cDNAs, they were recovered from the gel, purified with a gel cleanup kit, and subjected to sequencing reactions in the presence of fluorescentlabeled nucleotides, then analyzed by ABI Prism 377 DNA sequencer (Perkin-Elmer, Boston, MA, USA). All the obtained sequences corresponded to the expected ones (data not shown). The crossing points $\left(C_{\mathrm{p}}\right)$ for each reaction were determined and calculation of data was performed with the $\Delta \Delta C_{\mathrm{p}}$ method using the LightCycler relative quantification software 1.0 (Roche Diagnostics). Expression of the target genes in the tumoral thyroid tissues or tumor-derived cell lines was normalized respectively, against the expression found in the matched normal tissues or the HTU5 cells, and reported as fold of variation.

\section{Western blot analysis}

Cells protein extracts were obtained as previously described (Ulisse et al. 2006a). Aliquots of $50 \mu \mathrm{g}$ cell or tissue extracts were resolved on a $12.5 \%$ SDSPAGE and transferred onto nitrocellulose membranes. Incubations with anti-TACC3 (1:250) or anti-actin (1:500) primary antibodies were performed in $2.5 \%$ BSA in Tris buffer saline containing $0.05 \%$ of Tween 20 (TBST) at $4{ }^{\circ} \mathrm{C}$ overnight. Membranes were then incubated with anti-goat $(1: 30000)$ or anti-rabbit (1:50 000) horseradish peroxidase-conjugated secondary antibodies. The western blots were revealed by chemiluminescence using the Super Signal kit from Pierce (Rockford, IL, USA).

\section{Immunofluorescence}

The different cells lines were growth on glass cover slips and treated as described (Ulisse et al. 2006a). The cells were incubated for $1 \mathrm{~h}$ at room temperature with the anti-TACC3 antibody (1:100) and/or anti-AuroraA (1:200) and anti- $\gamma$-tubulin (1:200) or anti- $\beta$-tubulin (1:200) antibodies. After washing, the cover slips were incubated with a TRITC-conjugated anti-goat (1:100) and FITC-conjugated anti-mouse (1:100) antibodies for $1 \mathrm{~h}$ at room temperature and then mounted in Vectashield (Vector Laboratories, Burlingame, CA, USA) containing $1 \mu \mathrm{g} / \mathrm{ml}$ DAPI. Cover slips were observed with a microscope Leica-DMRXA.

\section{Co-immunoprecipitation of Aurora-A and TACC3}

Five microliters of dried Sepharose protein-G (4 Fastflow Sepharose, Amersham) were washed with $500 \mu \mathrm{l}$ lysis buffer and treated for $1 \mathrm{~h}$ at $4{ }^{\circ} \mathrm{C}$ with $500 \mu \mathrm{l}$ lysis buffer added with 5\% BSA. After five washes, the beads were incubated with $50 \mu \mathrm{l}$ antibody anti-AuroraA or anti-TACC3 (sc-22773) for $2 \mathrm{~h}$ at $4{ }^{\circ} \mathrm{C}$, and then washed twice with $500 \mu \mathrm{l}$ PBS. Beads were then incubated with a $2.5 \times 10^{6}$ cells extract for $2 \mathrm{~h}$ at $4{ }^{\circ} \mathrm{C}$ on a wheel. The beads were then washed five times with $500 \mu \mathrm{l}$ lysis buffer. Bound proteins were eluted in 
$10 \mu \mathrm{l}$ of $2 \mathrm{X}$-Laemmli sample buffer and the proteins were separated on a $12.5 \%$ SDS-PAGE and then immunoblotted. The western blot analyses were performed with the anti-TACC3 (1:250) and antiAurora-A (1:200) antibodies.

\section{Cell cycle analysis}

The FTC-133 cells were cultured up to $70-80 \%$ of confluence and pulse-labeled with $30 \mathrm{mM}$ BrdU for $2 \mathrm{~h}$ at $37^{\circ} \mathrm{C}$. The cells were then rinsed with PBS and collected by scraping with a rubber policeman in PBS. Following centrifugation, the cells were fixed in ice-cold ethanol and FITC-PI stained as previously described (Chang et al. 1999). All cell samples were analyzed for DNA content (PI) and/or BrdU content (FITC) using an EPICS Elike Flow cytometer (Coultronics, Hialeah, FL, USA) equipped with an argon laser $(488 \mathrm{~nm})$. Data analysis was carried out using Multicycle software (Phoenix Flow Systems, San Diego, CA, USA).

\section{Statistical analysis}

All the results are expressed as the mean \pm s.E.M. of at least three independent experiments and values were statistically compared using the Student's $t$-test or the two-tailed Wilcoxon rank sum test. Correlation analysis between the fold of increase of TACC 3 and Aurora-A mRNAs was evaluated by the Pearson correlation test using the SPSS software (SPSS Inc., Chicago, IL, USA). The results were determined to be significantly different if $P$ values were lower than 0.05 .

\section{Results}

\section{Expression, cell cycle regulation, and localization of TACC3 in normal human thyrocytes (HTU5)}

We first evaluated the expression at the mRNA and protein level of the TACC 3 gene in HTU5 cells. We demonstrated the presence of a specific TACC 3 transcript in HTU5 cells. The omission of the reverse transcriptase (negative control) prevented the formation of amplicons (Fig. 1A). Western blot analysis of HTU5 cell protein extracts demonstrated the presence of an immunoreactive band of $\sim 100 \mathrm{kDa}$, which was completely abrogated when the antiTACC 3 antibody was preincubated with the immune peptide (Fig. 1B).

We next determined whether TACC 3 expression was cell cycle regulated in HTU5 cells. To this end, the cells were cultured for 4 days in the presence of full medium containing 5\% FBS or in medium containing low serum concentration $(0.3 \%)$. Some of the latter were then exposed to fresh medium containing 5\% FBS for $24 \mathrm{~h}$. In these conditions, we previously shown, by means of flow cytometer analysis, that serum deprivation for 4 days reduced the number of proliferating cells $(\mathrm{S}+\mathrm{G} 2 / \mathrm{M}$ phase) by more than $75 \%$, while the exposure to fresh medium containing 5\% FBS restored the number of proliferating cells to control level (Ulisse et al. 2006a). As shown in Fig. 1C and D, serum deprivation induced a marked reduction in the expression of TACC 3 gene, at both mRNA and protein level. The exposure to fresh full medium restored the expression of TACC 3 gene at control level (Fig. 1C and D). As TACC3 is cell cycle regulated in thyrocytes, we next investigated the subcellular localization of TACC 3 protein by indirect immunofluorescence microscopy in the HTU5 cells. In all interphasic cell lines tested, the TACC 3 immunoreactivity was localized around the nuclei and decorated part of the interphasic microtubules (Fig. 1E, upper panel). In mitotic cells, TACC3 localized on the spindle microtubules, where $\beta$-tubulin was also localized, and in the pericentrosomal material (PCM) area. No staining for TACC 3 protein was observed onto the centrosome, as demonstrated by the double staining with $\gamma$-tubulin (Fig. 1E, middle and lower panels).

\section{Interaction of TACC3 with Aurora-A in thyroid cells}

Since Aurora-A has been reported to interact with TACC3 in other cellular systems, we analyzed their interaction in thyroid cells. To this end, the FTCderived cell line, the FTC-133 was employed, since these cells express a high level of Aurora-A (Ulisse et al. 2006a) and, as described above, a relative high amount of TACC3. As in HTU5 cells, TACC 3 co-localized with $\beta$-tubulin, but not $\gamma$-tubulin on the spindle microtubules, and was observed on the PCM (Fig. 2A, upper and middle panels). As expected, Aurora-A was found to localize on the centrosome and the microtubules of the spindle poles (Fig. 2A, lower panel). TACC 3 co-localized with Aurora-A solely on the PCM and the microtubules of the spindle poles. We thus investigated the interaction between TACC3 and Aurora-A by immunoprecipitating Aurora-A or TACC 3 from FTC-133 cell extracts. The presence of TACC 3 in the Aurora-A immunoprecipitate as well as that of Aurora-A in the TACC 3 immunoprecipitate, demonstrated that Aurora-A and TACC 3 interact, either directly or indirectly, in human thyroid cells (Fig. 2B). 
A

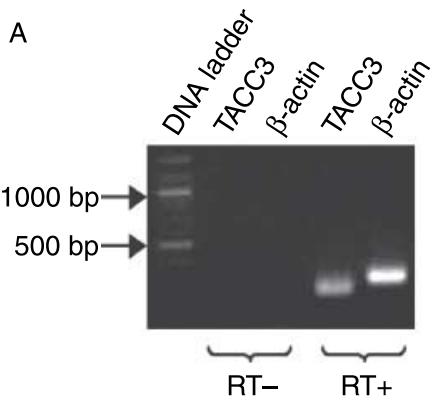

C

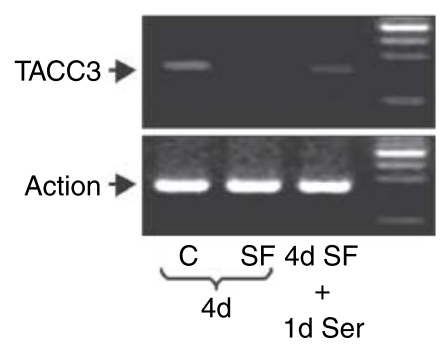

E

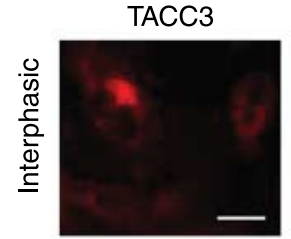

TACC3

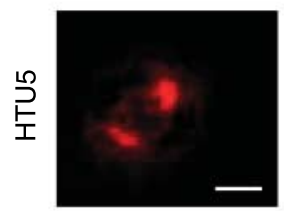

TACC3

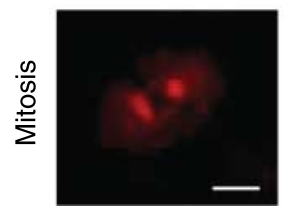

B

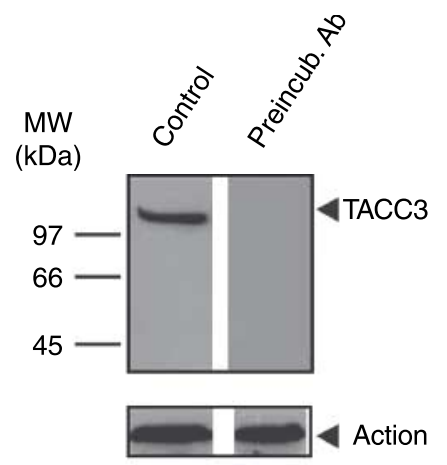

D MW
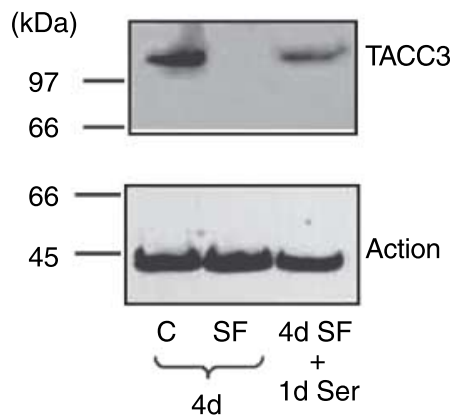

DAPI

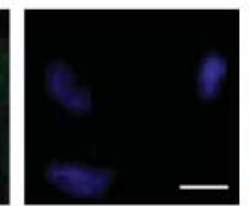

DAPI

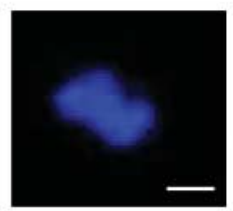

DAPI

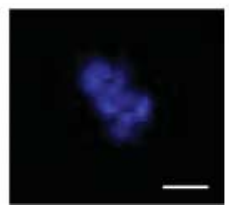

Merge

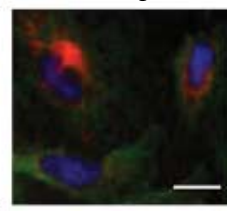

Merge

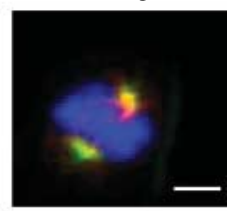

Merge

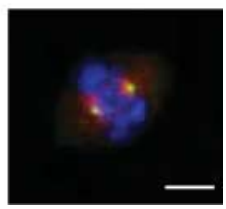

Figure 1 Expression, cell cycle regulation, and localization of TACC3 in normal human thyrocytes (HTU5). (A) Aliquots of $5 \mu \mathrm{g}$ DNA from HTU5 cells were used to prepare cDNA in the presence or absence of the reverse transcriptase. The cDNAs were amplified by quantitative RT-PCR using specific primers for TACC3 and actin as described in Materials and methods. (B) Fifty micrograms of cell protein extracts have been analyzed by western blot with an anti-TACC3 antibody (diluted 1/250) preincubated or not with the immunogenic peptide (upper panel) and anti-actin antibody (1/500; lower panel). (C and D) HTU5 cells have been treated for 4 days in the presence of $5 \%(C)$ or $0.3 \%$ (SF) FBS, some of the latter were then cultured for additional $24 \mathrm{~h}$ in media containing $5 \%$ FBS. Quantitative RT-PCR (C) and western blot (D) analyses for TACC3 were performed on cell extracts as described in Materials and methods. Data reported are representative of one out of three experiments. (E) Interphasic (upper panel) and mitotic (middle and lower panels) HTU5 cells were stained for TACC3 and $\beta$-tubulin (upper and middle panels) as a marker of microtubules or $\gamma$-tubulin (lower panel) as a marker for centrosome as described in Material and methods. Scale bar, $10 \mu \mathrm{m}$. 
A

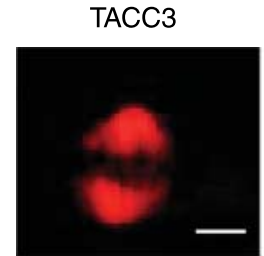

TACC3

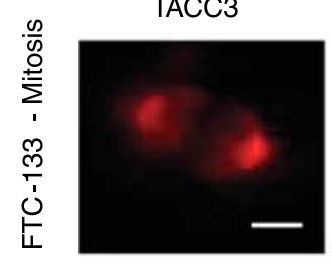

TACC3

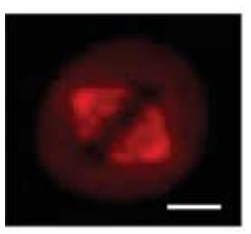

$\beta-T u b$

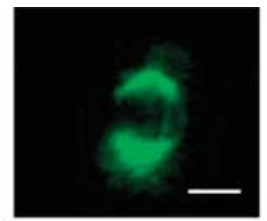

$\gamma-$ Tub

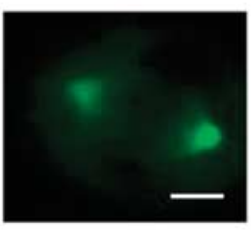

Aurora-A

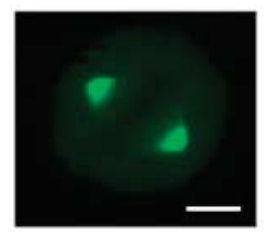

FTC-133

cell extracts

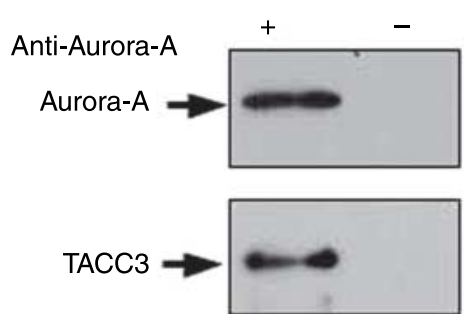

DAPI

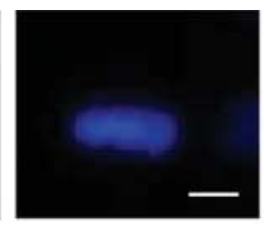

DAPI

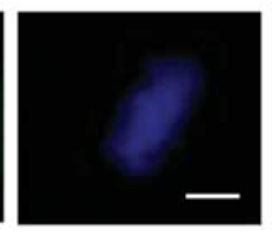

DAPI

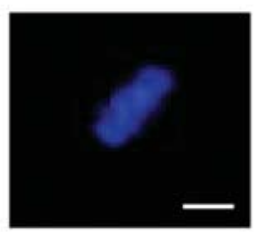

C

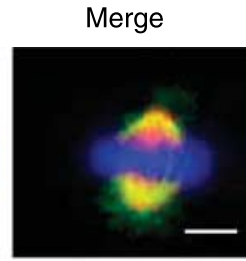

Merge

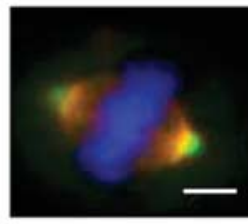

Merge

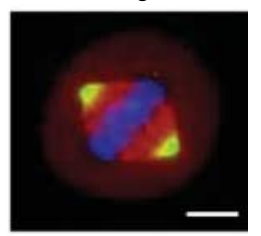

FTC-133

cell extracts

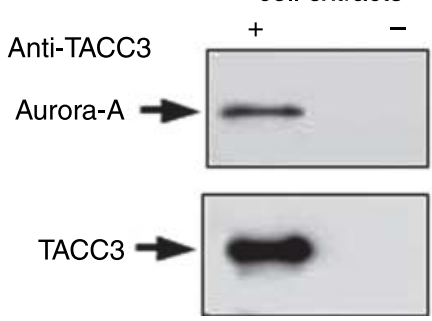

Figure 2 TACC3 and Aurora A kinase interaction and localization in FTC-133 thyroid cells. (A) Mitotic FTC-133 cells were stained for TACC3 and $\beta$-tubulin (upper panel) or $\gamma$-tubulin (middle panel) or Aurora-A (lower panel) as described in Materials and methods. Scale bar, $10 \mu \mathrm{m}$. (B and C) Aurora-A or TACC3 were immunoprecipitated from FTC-133 cell extracts using either the anti-Aurora-A monoclonal antibody (B) or the anti-TACC3 polyclonal antibody (C). Aurora-A and TACC3 were then immunodetected with the anti-Aurora-A monoclonal antibody (upper panel) and the polyclonal anti-TACC3 antibody (lower panel) as described in Materials and methods.

\section{Effects of VX-680 on FTC-133 cell ploidy, centro- some maturation, and TACC3 localization}

In the attempt to elucidate the functional role of Aurora-A and TACC 3 interaction, the effects of FTC133 treatment with the Aurora kinase inhibitor VX-680 were studied. In particular, FTC-133 cells were treated with VX-680 at $500 \mathrm{nM}$, a concentration previously shown on different cell types to elicit maximal response (Harrington et al. 2004). Analysis of cell DNA content after 24-h treatment, by flow cytometer analysis, showed the accumulation of cells with $\geq 4 \mathrm{~N}$ DNA content (Fig. 3A). Cell treatment with VX-680 did not affect neither the levels of TACC 3 or Aurora-A proteins (Fig. 3B). Immunofluorescence experiments demonstrated that $84.1 \%$ of VX-680-treated cells have more than two centrosomes compared with $4 \%$ in control cells (Fig. 3C). In the latter, all mitotic cells showed the presence of aberrant spindles characterized by shorter microtubules, or no spindle. In VX-680treated cells, Aurora-A still present on the centrosomes, while TACC 3 was missing on the spindle microtubules (Fig. 3C).

\section{TACC3 gene expression in transformed human thyrocytes}

The above observations lead us to investigate the expression level of TACC3 gene in different human cell lines derived from benign follicular adenoma (HTU42), follicular (FTC-133), papillary (B-CPAP), and anaplastic (8305C and CAL-62) thyroid carcinomas. Quantitative RT-PCR analysis revealed that TACC3 mRNA levels were similar in the HTU42 and HTU5 cells, while a lower 
A

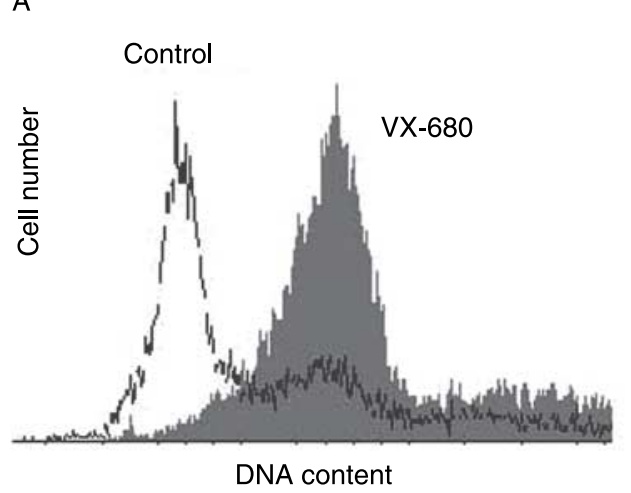

B
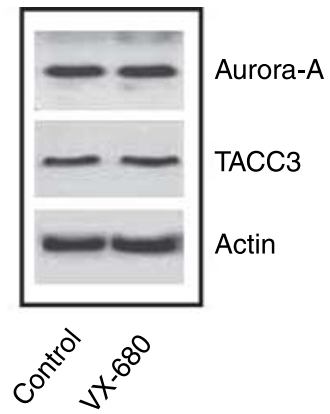

C

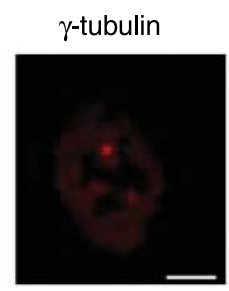

Aurora-A

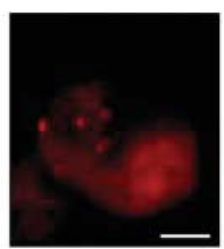

TACC3

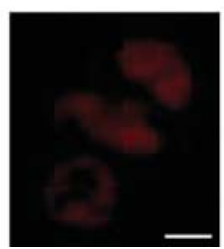

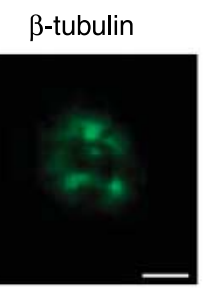

$\gamma$-tubulin

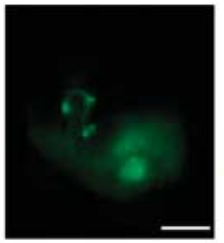

$\beta$-tubulin

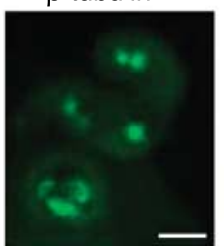

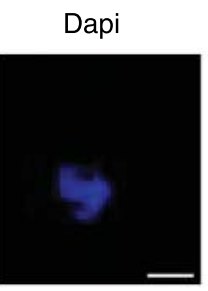

Dapi

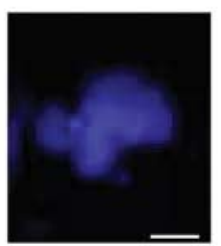

Dapi

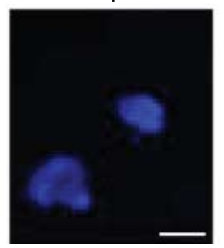

Merge

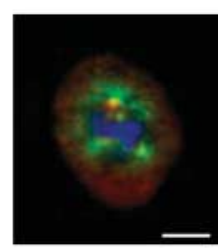

Merge

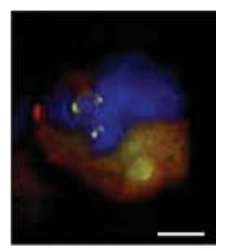

Merge

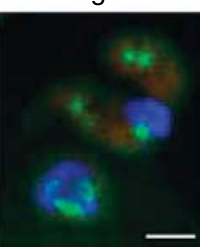

Figure 3 Effects of Aurora kinase inhibition by VX-680 on FTC-133 cell ploidy, spindle formation and TACC3 subcellular localization. (A) FTC-133 cells were incubated for $24 \mathrm{~h}$ with $500 \mathrm{nM} \mathrm{VX-680} \mathrm{or} \mathrm{DMSO} \mathrm{used} \mathrm{as} \mathrm{vehicle} \mathrm{(control)} \mathrm{and} \mathrm{pulse} \mathrm{labeled} \mathrm{with} \mathrm{BrdU} \mathrm{for}$ $2 \mathrm{~h}$. The cells were then fixed and analyzed by flow as described in Materials and methods. (B) Fifty micrograms of cell protein extracts from control and VX-680-treated cells were analyzed by western blot with an anti-TACC3 antibody (diluted 1/250), antiAurora-A (diluted 1:500), or anti-actin antibody (1/500). (C) Subcellular localization of Aurora-A and TACC3 in control and VX-680treated FTC-133 mitotic cells. Fixed cells were stained for TACC3 and $\beta$-tubulin or Aurora-A and $\beta$-tubulin as described in Materials and methods. Scale bar, $10 \mu \mathrm{m}$.

expression was observed in all the carcinoma-derived cell lines (Fig. 4A). In particular, the TACC3 mRNA level was reduced in the FTC-133 $(0.72 \pm 0.06 ; P<0.01)$, the B-CPAP $(0.64 \pm 0.01 ; P<0.01)$, the $8305 \mathrm{C}(0.39 \pm$ $0.04 ; P<0.01)$, and the CAL-62 $(0.33 \pm 0.03 ; P<0.01)$ cell lines. Likewise, with respect to the HTU5 cells, the TACC 3 protein was found significantly reduced in the FTC-133 $(0.85 \pm 0.05 ; P<0.05)$, B-CPAP $(0.53 \pm 0.05$; $P<0.01), 8305 \mathrm{C}(0.35 \pm 0.13 ; P<0.01)$, and CAL-62 $(0.17 \pm 0.08 ; P<0.01)$, but similar in the HTU42 cells $(0.98 \pm 0.29$; Fig. 4B and C).

\section{Expression of TACC3 and Aurora-A in normal and differentiated thyroid carcinoma tissues}

The levels of the TACC 3 mRNA in PTC and FTC carcinoma tissues were compared with those of the matched normal thyroid by means of quantitative RT-PCR. The results are shown in Fig. 5A. TACC3 mRNA levels were lower in 7 out of 13 PTCs and 2 out of 3 FTCs, but higher in 6 PTCs and 1 FTC than in the normal tissue. Altogether, TACC 3 mRNA levels were reduced in $56 \%$ of the differentiated thyroid cancers 


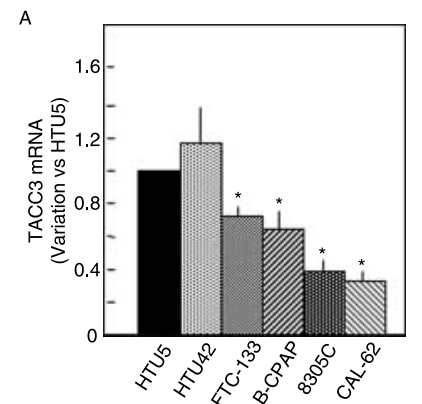

B

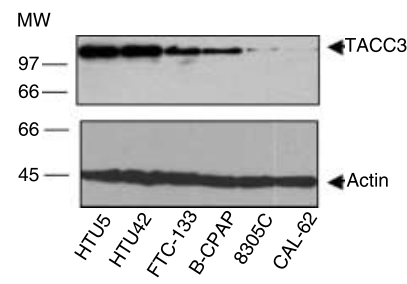

C

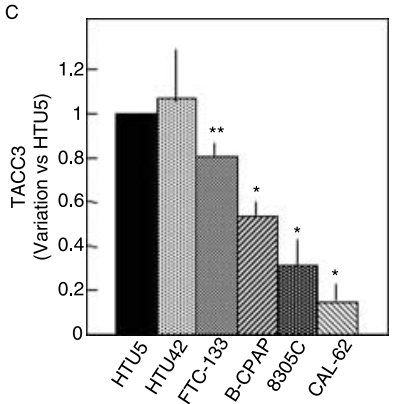

Figure 4 Expression of TACC 3 gene in normal human thyrocytes (HTU5) and cell lines derived from thyroid benign follicular adenoma (HTU42) and from papillary (B-CPAP), follicular (FTC-133), and anaplastic (8305C and CAL-62) thyroid carcinomas. (A) Quantitative RT-PCR analysis of TACC3 mRNA levels. Aliquots of $5 \mu \mathrm{g}$ RNA were analyzed by RT-PCR. Messenger RNA variations were relative to the TACC3/actin ratio observed in HTU5 cells. Data reported represent the mean \pm S.E.M. of three independent experiments. Statistical significance of data was assessed by Student's $t$-test. ${ }^{\star} P<0.01,{ }^{\star \star} P<0.05$. (B and C) Western blot analysis of TACC3 protein in the different cell lines as described above. Fifty micrograms of the cell protein extracts were analyzed for TACC3 and actin contents by immunoblotting (B). TACC3 variations $(C)$ were relative to the TACC3/actin ratio observed in HTU5 cells. Data reported represent the mean \pm s.E.M. of three independent experiments. Statistical significance of data was assessed by Student's $t$-test. ${ }^{*} P<0.01,{ }^{\star \star} P<0.05$.

(DTC; $0.50 \pm 0.07 ; P<0.01$ ) and increased in $44 \%$ of DTC $(1.96 \pm 0.35 ; P<0.05)$ when compared with their normal matched tissues. Since TACC 3 and Aurora-A interact in thyroid cells, the expression of Aurora-A was determined in the same tissues. The results are shown in Fig. 5B. Quantitative RT-PCR revealed that Aurora-A was upregulated in 5 out of 13 PTCs $(2.75 \pm 0.44$; $P<0.01)$. Downregulation of Aurora-A was noted in five PTCs $(0.51 \pm 0.13 ; P<0.01)$ and all three FTCs $(0.41 \pm 0.03 ; P<0.01)$.
Using a linear regression analysis, we evidenced a significant correlation $(r=0.717 ; P<0.01)$ between the variations of TACC 3 and Aurora-A mRNAs in thyroid cancer tissues (Fig. 5C). We finally attempted to correlate the variation of TACC3 and Aurora-A expression in thyroid cancer tissues with the clinical and histological parameters, including patient's age, stage, tumor size, and histology. No correlation could be found with any of the parameters analyzed.

\section{Discussion}

As our knowledge about the molecular process controlling cell division is growing, an increasing number of proteins are now suspected to play a relevant role in the genetic instability, an hallmark of solid cancers progression. Thyroid follicular neoplasms are often characterized by aneuploidy (Shahedian et al. 2001, Ouyang et al. 2002). In this context, we recently demonstrated that the three members of the Aurora kinase family, Aurora-A, Aurora-B, and Aurora-C, involved in the regulation of multiple aspects of chromosome segregation and cytokinesis, are overexpressed in malignant thyroid tumor-derived cell lines and tissues (Ulisse et al. 2006a). In particular, the alteration in Aurora-A gene expression may help to shed some light on the molecular mechanisms involved in the genetic instability of thyroid cancers. In fact, the overexpression of Aurora-A in different human cell lines has been shown to induce centrosome amplification and to potentiate the oncogenic action of Ras. In human thyrocytes, Ras participates in transformation and chromosome instability (Bischoff \& Plowman 1999, Tatsuka et al. 2005). Furthermore, it has been documented that p53 is a substrate of Aurora-A and its phosphorylation on Ser215 leads to the inhibition of the p53 transactivating action on several genes (Liu et al. 2004). Thus, alterations in the crosstalk between Aurora kinase A and p53 could be relevant in thyroid tumor progression by compromising the fidelity of chromosome segregation (Parry et al. 1998, Shahedian et al. 2001).

In order to get more insight about the molecular mechanism of thyroid cancer progression, we have here characterized, in normal and tumoral human thyrocytes and tissues, the expression of TACC 3 gene which encodes for a protein substrate of Aurora-A. TACC proteins belong to a conserved family of centrosome and microtubule-associated proteins whose functions are not clearly understood. Nevertheless, it is well established that these proteins are all involved in the regulation of the mitotic phase of the 
A

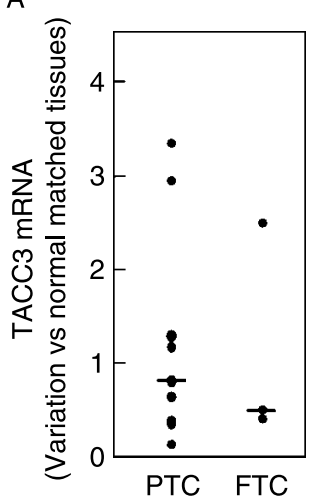

B

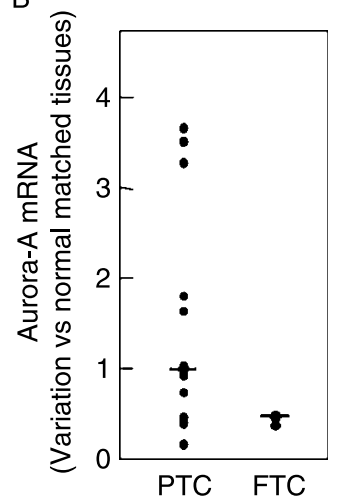

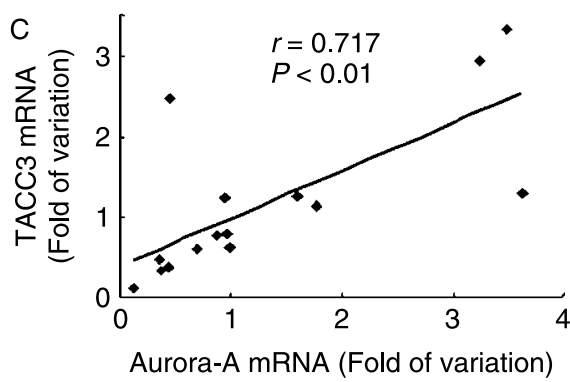

Figure 5 TACC 3 and Aurora-A kinase expression in matched cancer and normal thyroid tissues. (A and B) Quantitative RT-PCR analysis of TACC3 (A) and Aurora-A kinase (B) mRNA levels in 13 papillary (PTC) and 3 follicular (FTC) thyroid cancer tissues compared with normal matched tissues obtained from the same patients. mRNA variations were relative to the TACC3/actin or Aurora A/actin ratios observed in normal thyroid tissues. The small bars represent the median values. (C) Correlation analysis between the variations of TACC 3 and Aurora-A kinase mRNAs in the differentiated thyroid carcinomas (13 PTCs and 3 FTCs) analyzed.

cell cycle (Gergely 2002). Clues about their functions have come from Drosophila and Xenopus. TACC proteins are likely important in M-phase entry since they regulate both spindle assembly and mRNA translation (Pascreau et al. 2005, Peset et al. 2005). The three human TACC proteins are also suspected to play a role in oncogenesis. Aberrations of TACC genes have been associated with various cancers (Still et al. 1999a, Conte et al. 2002, Line et al. 2002, Lauffart et al. 2005). In particular, TACC3 have been found overexpressed in various cancer cell lines (Still et al. $1999 a$ ) but, in contrast, lost in $67 \%$ ovarian tumors (Lauffart et al. 2005).

Up to now, the expression and functions of TACC3 in human thyrocytes have not been documented. In the present report, we demonstrate that TACC3 gene is expressed in normal human thyrocytes. Its expression is likely cell cycle regulated, since it is downregulated in serum-deprived cultured cells and restored after serum addition. Our immunofluorescence results

showing that TACC 3 is localized along the spindle microtubules in mitotic cells are in favor of a cell cycle-regulated expression of the TACC 3 protein in thyrocytes.

In Xenopus and Drosophila, Aurora-A has been shown to interact with the human TACC 3 orthologues, maskin, and D-TACC respectively (Giet et al. 2002, Pascreau et al. 2005). In human thyroid cells, Aurora-A and TACC 3 co-localized at the spindle pole, where they may have a role in microtubules growth and stability, as suggested in Drosophila (Raff 2002). Inhibition of Aurora-A by VX-680 in FTC-133 cells which lead to abnormal spindle formation characterized by shorter microtubules, delocalized TACC 3 from the spindle microtubules. This suggests that the kinase activity of Aurora-A is needed for the localization of TACC 3 on the spindle. However, even in the presence of VX-680, Aurora-A and TACC3 co-immunoprecipitated (data not shown). The apparent discrepancy between the IP and the IF observation in VX-680-treated cells may be due to the fact that the VX-680 is a reversible inhibitor and that reassociation of the two proteins may take place during the cell protein extraction procedure used for the co-immunoprecipitation experiments. Nevertheless, these observations suggest that Aurora-A activity is, directly or indirectly, required for TACC 3 localization on the spindle microtubule. This is in agreement with recent observations in Xenopus laevis egg extracts showing that Aurora-A phosphorylation of TACC3/ maskin is required for centrosome-dependent microtubule assembly in mitosis (Kinoshita et al. 2005).

Opposite variations of TACC expression have been reported in various cancers. Upregulation of TACC3 expression has been observed during the transition of breast cancer from in situ preinvasive ductal carcinoma to invasive ductal carcinoma, and in multiple myeloma (Still et al. 1999a, Ma et al. 2003). In contrast, a high proportion of human breast and ovarian cancer tissues shows a downregulation of TACC 1 and TACC 3 expression (Conte et al. 2002, 2003, Lauffart et al. 2005). In this work, we show that TACC 3 expression was significantly downregulated in different thyroid carcinoma-derived cell lines, but not in a cell line derived from a benign follicular thyroid tumor. In particular, the reduction of TACC 3 expression was more important in the two cell lines derived from the highly aggressive human anaplastic thyroid carcinomas (8305C and CAL-62). In contrast, in samples of differentiated thyroid cancer (DTC) tissues, both upand downregulation were observed. Fifty-six percent of DTC showed a significant reduction in TACC 3 gene expression while TACC 3 expression was found to be 
significantly upregulated in $44 \%$. Taken all together, the findings reported here may suggest that, in thyroid cells, TACC3 protein may function as both transforming and tumor-suppressor factor, as demonstrated in other cell types (Chen et al. 2000, Raff 2002).

Since TACC 3 and Aurora-A are partners in thyroid cells, the expression of Aurora-A was analyzed in the same tissues. As for TACC 3, both up- and downregulation of Aurora-A was observed. In a recent study, performed on seven normal matched PTC tissues, we reported an upregulation of Aurora-A in five over seven normal matched PTC tissues (Ulisse et al. 2006a). In the new series of thyroid cancer tissues analyzed here, we found that Aurora-A expression was upregulated in 5 out of 13 PTC tissues, unchanged in three and downregulated in five PTC as well as in three FTC tissues. Thus, we may speculate that, as hypothesized for TACC3, either up- or downregulation of Aurora-A may lead to a proliferative advantage for thyroid cancer cells. This is not surprising since both the up- and downregulation of its expression have been reported to cause abnormal mitosis with defects in chromosome segregation and cytokinesis (Bischoff \& Plowman 1999). Interestingly, we found that the expression of TACC 3 and Aurora-A in thyroid cancer varied together. This observation corroborates similar findings reported in breast cancer tissues (Conte et al. 2003) and suggests common molecular mechanism(s) regulating their expression. In breast cancers, increased expressions of TACC 3 and Aurora-A mRNAs have been linked with cancer progression and high-grade tumors (Ma et al. 2003). In contrast, in the series of thyroid cancers analyzed here, no correlation was found between the expression of either Aurora-A or TACC 3 , and clinical or histological parameters (including patient's age, tumor stage, size, or histology). Nevertheless, this needs to be confirmed on a larger number of cases.

In conclusion, we demonstrated that TACC 3 gene is expressed in human thyroid cells in a cell cycle-related manner. In human thyrocytes, TACC3 interacted with Aurora-A, whose activity is required for its localization on the spindle microtubules. We also observed that TACC 3 and Aurora-A expression varied together in thyroid cancer tissues. Altogether, this suggests that these proteins may serve similar cellular functions and that deregulation of one or both gene expression may participate to thyroid cancer aneuploidy.

\section{Acknowledgements}

This work was supported by the Ministero Italiano dell'Università e della Ricerca (PRIN 2004), the
Agenzia Spaziale Italiana, the Ligue Nationale Contre le Cancer, and the Association pour la Recherche sur le Cancer (ARC). The authors declare that there is no conflict of interest that would prejudice the impartiality of this scientific work.

\section{References}

Arlot-Bonnemains Y \& Prigent C 2002 Cell cycle. A trigger for centrosome duplication. Science 295 455-456.

Bischoff JR \& Plowman GD 1999 The Aurora/Ipl1p kinase family: regulators of chromosome segregation and cytokinesis. Trends in Cell Biology 9 454-459.

Carmena M \& Earnshaw WC 2003 The cellular geography of aurora kinases. Nature Reviews Molecular Cell Biology 4 842-854.

Chang D, Chen F, Zhang F, McKay BC \& Ljungman M 1999 Dose-dependent effects of DNA-damaging agents on p53mediated cell cycle arrest. Cell Growth and Differentiation 10 155-162.

Chen HM, Schmeichel KL, Mian IS, Lelievre S, Petersen OW \& Bissell MJ 2000 AZU-1: a candidate breast tumor suppressor and biomarker for tumor progression. Molecular Biology of the Cell 11 1357-1367.

Chomczynski P \& Sacchi N 1987 Single-step method of RNA isolation by acid guanidinium thiocyanate-phenolchloroform extraction. Analytical Biochemistry 162 156-159.

Conte N, Charafe-Jauffret E, Delaval B, Adelaide J, Ginestier C, Geneix J, Isnardon D, Jacquemier J \& Birnbaum D 2002 Carcinogenesis and translational controls: TACC 1 is down-regulated in human cancers and associates with mRNA regulators. Oncogene 21 5619-5630.

Conte N, Delaval B, Ginestier C, Ferrand A, Isnardon D, Larroque C, Prigent C, Seraphin B, Jacquemier J \& Birnbaum D 2003 TACC1-chTOG-Aurora A protein complex in breast cancer. Oncogene 22 8102-8116.

Curcio F, Ambesi-Impiombato FS, Perrella G \& Coon HG 1994 Long-term culture and functional characterization of follicular cells from adult normal human thyroids. PNAS 91 9004-9008.

Gergely F 2002 Centrosomal TACCtics. Bioessays 24 915-925.

Gergely F, Karlsson C, Still I, Cowell J, Kilmartin J \& Raff JW 2000 The TACC domain identifies a family of centrosomal proteins that can interact with microtubules. PNAS 97 14352-14357.

Giet R, McLean D, Descamps S, Lee MJ, Raff JW, Prigent C \& Glover DM 2002 Drosophila Aurora A kinase is required to localize D-TACC to centrosomes and to regulate astral microtubules. Journal of Cell Biology 156 437-451.

Harrington EA, Bebbington D, Moore J, Rasmussen RK, Ajose-Adeogun AO, Nakayama T, Graham JA, Demur C, Hercend T, Diu-Hercend A et al. 2004 VX-680, a potent and selective small molecule inhibitor of the Aurora kinases, suppresses tumor growth in vivo. Nature Medicine 10 262-267. 
Kinder BK 2003 Well differentiated thyroid cancer. Current Opinion in Oncology 15 71-77.

Kinoshita K, Noetzel TL, Pelletier L, Mechtler K, Drechsel DN, Schwager A, Lee M, Raff JW \& Hyman AA 2005 Aurora A phosphorylation of TACC $3 /$ maskin is required for centrosome-dependent microtubule assembly in mitosis. Journal of Cell Biology 170 1047-1055.

Lauffart B, Gangisetty O \& Still IH 2003 Molecular cloning, genomic structure and interactions of the putative breast tumor suppressor TACC2. Genomics 81 192-201.

Lauffart B, Vaughan MM, Eddy R, Chervinsky D, Dicioccio RA, Black JD \& Still IH 2005 Aberrations of TACC1 and TACC3 are associated with ovarian cancer. $B M C$ Women's Health 58.

Line A, Slucka Z, Stengrevics A, Li G \& Rees RC 2002 Altered splicing pattern of TACC1 mRNA in gastric cancer. Cancer Genetics and Cytogenetics 139 78-83.

Liu Q, Kaneko S, Yang L, Feldman RI, Nicosia SV, Chen J \& Cheng JQ 2004 Aurora-A abrogation of p53 DNA binding and transactivation activity by phosphorylation of serine 215. Journal of Biolgical Chemistry 279 52175-52182.

Ma XJ, Salunga R, Tuggle JT, Gaudet J, Enright E, McQuary P, Payette T, Pistone M, Stecker K, Zhang BM et al. 2003 Gene expression profiles of human breast cancer progression. PNAS 100 5974-5979.

Nikiforova MN, Lynch RA, Biddinger PW, Alexander EK, Dorn GW II, Tallini G, Kroll TG \& Nikiforov YE 2003 RAS point mutations and PAX8-PPAR gamma rearrangement in thyroid tumors: evidence for distinct molecular pathways in thyroid follicular carcinoma. Journal of Clinical Endocrinology and Metabolism 88 2318-2326.

Ouyang B, Knauf JA, Ain K, Nacev B \& Fagin JA 2002 Mechanisms of aneuploidy in thyroid cancer cell lines and tissues: evidence for mitotic checkpoint dysfunction without mutations in BUB1 and BUBR1. Clinical Endocrinology 56 341-350.

Parry EM, Ulucan H, Wyllie FS, Wynford-Thomas D \& Parry JM 1998 Segregational fidelity of chromosomes in human thyroid tumour cells. Chromosoma 107 491-497.

Pascreau G, Delcros JG, Cremet JY, Prigent C \& ArlotBonnemains Y 2005 Phosphorylation of maskin by Aurora-A participates in the control of sequential protein synthesis during Xenopus laevis oocyte maturation. Journal of Biolgical Chemistry 280 13415-13423.

Pasieka JL 2003 Anaplastic thyroid cancer. Current Opinion in Oncology 15 78-83.

Perrella G, Fabbro D, Damante G, Di Loreto C, Beltrami CA, Curcio F \& Ambesi-Impiombato FS 1997 Expression of differentiation markers in cultured cells from various thyroid diseases. Thyroid 7 817-821.

Peset I, Seiler J, Sardon T, Bejarano LA, Rybina S \& Vernos I 2005 Function and regulation of Maskin, a TACC family protein, in microtubule growth during mitosis. Journal of Cell Biology 170 1057-1066.
Piekorz RP, Hoffmeyer A, Duntsch CD, McKay C, Nakajima H, Sexl V, Snyder L, Rehg J \& Ihle JN 2002 The centrosomal protein TACC3 is essential for hematopoietic stem cell function and genetically interfaces with p53regulated apoptosis. EMBO Journal 21 653-664.

Raff JW 2002 Centrosomes and cancer: lessons from a TACC. Trends in Cell Biology 12 222-225.

Sadek CM, Jalaguier S, Feeney EP, Aitola M, Damdimopoulos AE, Pelto-Huikko M \& Gustafsson JA 2000 Isolation and characterization of AINT: a novel ARNT interacting protein expressed during murine embryonic development. Mechanism of Development 97 13-26.

Sadek CM, Pelto-Huikko M, Tujague M, Steffensen KR, Wennerholm M \& Gustafsson JA 2003 TACC3 expression is tightly regulated during early differentiation. Gene Expression Patterns 3 203-211.

Sato T, Saito H, Morita R, Koi S, Lee JH \& Nakamura Y 1991 Allelotype of human ovarian cancer. Cancer Research 51 5118-5122.

Shahedian B, Shi Y, Zou M \& Farid NR 2001 Thyroid carcinoma is characterized by genomic instability: evidence from p53 mutations. Molecular Genetics and Metabolism 72 155-163.

Sherman SI 2003 Thyroid carcinoma. Lancet 361 501-511.

Still IH, Vince P \& Cowell JK $1999 a$ The third member of the transforming acidic coiled coil-containing gene family, TACC 3, maps in 4p16, close to translocation breakpoints in multiple myeloma, and is upregulated in various cancer cell lines. Genomics 58 165-170.

Still IH, Hamilton M, Vince P, Wolfman A \& Cowell JK $1999 \mathrm{~b}$ Cloning of TACC1, an embryonically expressed, potentially transforming coiled coil containing gene, from the 8 p11 breast cancer amplicon. Oncogene 18 4032-4038.

Still IH, Vettaikkorumakankauv AK, DiMatteo A \& Liang P 2004 Structure-function evolution of the transforming acidic coiled coil genes revealed by analysis of phylogenetically diverse organisms. BMC Evolutionary Biology 416.

Tatsuka M, Sato S, Kitajima S, Suto S, Kawai H, Miyauchi M, Ogawa I, Maeda M, Ota T \& Takata T 2005 Overexpression of Aurora-A potentiates HRAS-mediated oncogenic transformation and is implicated in oral carcinogenesis. Oncogene 24 1122-1127.

Ulisse S, Delcros JG, Baldini E, Toller M, Curcio F, Giacomelli L, Prigent C, Ambesi-Impiombato FS, D’Armiento M \& Arlot-Bonnemains Y $2006 a$ Expression of Aurora kinases in human thyroid carcinoma cell lines and tissues. International Journal of Cancer 119 275-282.

Ulisse S, Baldini E, Toller M, Marchioni E, Giacomelli L, De Antoni E, Ferretti E, Marzullo A, Gracianno F, Trimoli P et al. $2006 b$ Diffenretial expression of the components of the plaminogen actviating system in human thyroid tumor derived cell lines and papillary carcinomas. European Journal of Cancer 42 2631-2638. 